\title{
Mitral Annulus Segmentation using Deep Learning in 3D Transesophageal Echocardiography
}

\author{
Børge Solli Andreassen, Federico Veronesi, Olivier Gerard, Anne H. Schistad Solberg, Eigil Samset
}

\begin{abstract}
D Transesophageal Echocardiography is an excellent tool for evaluating the mitral valve and is also well suited for guiding cardiac interventions. We introduce a fully automatic method for mitral annulus segmentation in 3D Transesophageal Echocardiography, which requires no manual input. One hundred eleven multi-frame 3D transesophageal echocardiography recordings were split into training, validation, and test sets. Each 3D recording was decomposed into a set of 2D planes, exploiting the symmetry around the centerline of the left ventricle. A deep 2D convolutional neural network was trained to predict the mitral annulus coordinates, and the predictions from neighboring planes were regularized by enforcing continuity around the annulus. Applying the final model and postprocessing to the test set data gave a mean error of 2.0 $\mathrm{mm}$ - with a standard deviation of $1.9 \mathrm{~mm}$. Fully automatic segmentation of the mitral annulus can alleviate the need for manual interaction in the quantification of an array of mitral annular parameters and has the potential to eliminate inter-observer variability.
\end{abstract}

Index Terms-Deep learning, echocardiography, machine learning, mitral annulus segmentation, soft-argmax

\section{INTRODUCTION}

$\mathbf{T}$ HE mitral apparatus comprises two leaflets and a saddleshaped annulus located between the left ventricle and the left atrium of the heart [1]. The valve functions as a seal, preventing blood from flowing back into the atrium when the ventricle contracts [1]. Mitral stenosis is a common complication of rheumatic fever, with high prevalence in underdeveloped countries [2] [3]. While valvular diseases are relatively infrequent in industrialized countries, the prevalence increases with age - affecting more than $10 \%$ of the population above 75 years of age, with mitral regurgitation being the most common disease [4].

Submitted for review May 31st, 2019; revised November 27th, 2019; accepted December 7th, 2019.

B. S. Andreassen is with the Department of Informatics, University of Oslo, 0316 Oslo, Norway (e-mail: borgesan@ifi.uio.no).

F. Veronesi, PhD, is with GE Vingmed Ultrasound. 3183 Horten, Norway. (email: federico.veronesi@ge.com)

O. Gerard, PhD, is with GE Vingmed Ultrasound. 3183 Horten, Norway. (email: olivier.gerard@ge.com)

Prof. A. Solberg is with the Department of Informatics, University of Oslo, 0316 Oslo, Norway (e-mail: anne@ifi.uio.no).

Prof. E. Samset is with GE Vingmed Ultrasound, Center for Cardiological Innovation and Department of Informatics, University of Oslo, 0316 Oslo, Norway (e-mail: eigil.samset@ge.com).
Echocardiography is the most frequently used imaging modality to evaluate mitral valve diseases [5]. Transesophageal Echocardiography (TEE) is the recommended echocardiographic imaging modality for assessing valvular diseases [6]. During TEE examinations, the probe is located inside the esophagus. Studies have found 3D TEE to be superior to 2D TEE in localizing pathologies related to mitral regurgitation [7]-[9]. TEE is also well suited for guiding cardiac interventions [10].

Localization of the mitral annulus and leaflets is the first step in order to calculate clinical measures required for both diagnosing pathologies and planning interventions of the mitral apparatus [11]. Due to its clinical importance, localization of the mitral annulus in echocardiography has been the subject of both academic research and the focus of commercial development [12]-[15]. Several works have introduced semiautomatic methods using both classical computer vision [16], [17] and machine learning [12], [18] approaches - often augmented with anatomy-based priors.

Schneider et al. [16] presented a semi-automatic method for segmenting the mitral annulus in 3D volumes of the ventricular systole. Given one input point on the mitral leaflet, they defined a thin tissue detector (by applying convolution with a Gaussian kernel) that detects a coarse segmentation of the mitral leaflets. Using graph-cuts and maximum flow, they estimated the annulus of the mitral valve. In [17], Schneider $e t$ al. extended the method from [16] using optical flow to cover the entire cardiac cycle.

Tiwari et al. [18] utilized the thin tissue detector presented in [16] and applied a Naive Bayes classifier to predict the mitral annulus in radial 2D planes. Common for all these methods is that they require manual input of anatomical landmarks.

Ionasec et al. [12] introduced a fully automatic, patientspecific method for modeling and quantifying the aortic and mitral valves in both 4D TEE and cardiac CT. The method combined physiological models with several machine learning methods - including marginal space learning [19], probabilistic boosting-trees, and an algorithm called trajectory spectrum learning. Pedrosa et al. [20] used optical flow and active surfaces for a fully automatic mitral valve segmentation in 4D Transthoracic Echocardiograms.

We present a fully automatic deep learning based method for mitral annulus segmentation in 3D TEE images. By taking 
advantage of the symmetry of the problem, predictions are made on 2D images. We use angular momentum as a proxy of confidence and reconstruct 3D predictions by constraining the 2D heatmaps - iteratively building the 3D curve segmentation. The problem is simplified as we take advantage of symmetry. The 2D approach reduces the computational requirement compared to a full $3 \mathrm{D} \mathrm{CNN}$, and increases the ratio of training data to the number of $3 \mathrm{D}$ volumes.

\section{Methodology}

\section{A. Patient data and annotation}

We collected 4D TEE acquisitions from 89 different - fully anonymized - patient examinations, from three hospitals. The images were acquired by certified physicians and sonographers using GE Vivid E9 and GE Vivid E95 scanners (GE Vingmed Ultrasound, Horten, Norway). Midesophageal level acquisitions with the mitral valve centered in the field of view a standard view in TEE examinations [21] - were selected for our experiments, 111 in total. In this view, the centerline of the left ventricle approximately coincides with the centerline of the volume. Acquisitions with serious artifacts, e.g., stitching artifacts [22], were excluded. Figure 1 depicts two planes the long axis and mitral commissural view — from one of the acquisitions.

Each acquisition contained one cineloop, with framerates between 4.7 to 66 frames per second and depths between $4.9 \mathrm{~cm}$ to $18 \mathrm{~cm}$. Full volume acquisitions were cropped at $9 \mathrm{~cm}$ depth, as the method assumes mitral valve focused acquisitions. For each recording, we used several 3D frames in the ventricular systole, i.e., frames with a closed mitral valve.

The annotations were performed by the first author using a commercially available medical software called 4D AutoMVQ (GE Vingmed Ultrasound, Horten, Norway) - a semiautomatic tool for segmentation of the mitral valve. This work was done based on instructions and guidance from a trained cardiology expert. The tool uses template matching of an a priori 3D saddle-shaped curve. Segmentation in 4D AutoMVQ required initialization of six anatomical points and fine tuning of the result. Fine tuning the segmentation at a point affects neighboring points with a Gaussian weighting. After fine-tuning of the segmentations, the annulus coordinates were exported as 58 Cartesian coordinates for each 3D volume.

\section{B. Data structure and labels}

Let $w_{x}, w_{y}$, and $w_{d}$ be the azimuth, elevation, and depth of a given $3 \mathrm{D}$ volume, and let the origin be defined at the ultrasound probe. The data volume lies inside:

$$
(x, y, z) \text { with }\left\{\begin{array}{l}
x \in\left[-w_{x} / 2, w_{x} / 2\right], \\
y \in\left[-w_{y} / 2, w_{y} / 2\right], \\
z \in\left[0, w_{d}\right] .
\end{array}\right.
$$

To standardize the field of view, each volume was cropped to a maximum of $9 \mathrm{~cm}$ depth. The method utilizes image
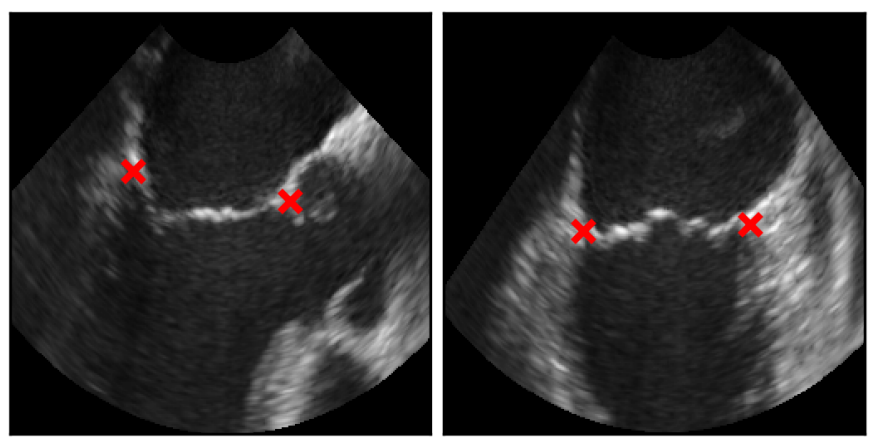

Fig. 1. Long axis (left) and mitral commissural (right) view from one of the 3D TEE images volumes in the dataset. The four points (red crosses) show the intersection between the mitral annulus and plane. The aim of our method is to automatically predict the annulus in the entire volume.
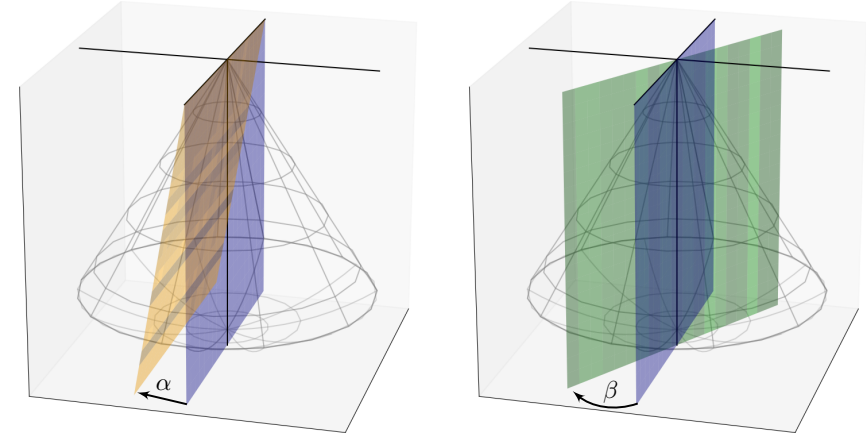

Fig. 2. Planes, $\mathbf{X}^{\boldsymbol{\alpha}, \boldsymbol{\beta}}$ (3) , generated as described in Section II-B. The mesh grid illustrates the ultrasound probe's field of view. The blue plane corresponds to the reference plane, $\mathrm{X}^{\mathbf{0}, 0}$, in both images. Changing $\alpha$ rotates the plane off the $z$-axis, as illustrated by the orange plane (left figure) with $\alpha=10^{\circ}$. Changing $\beta$ rotates the plane around the $z$-axis, as illustrated by the green plane (right figure) with $\beta=30^{\circ}$.

planes rotated around the $z$-axis of the volume. The planes were defined relative to the following reference plane segment:

$$
\mathbf{X}^{0,0}=\left\{(x, y, z) \mid x \in\left[-\frac{w_{x}}{2}, \frac{w_{x}}{2}\right], y=0, z \in\left[0, w_{d}\right]\right\} \text {. }
$$

Let $\mathbf{X}^{\alpha, \beta}$ be the plane segment resulting from:

1) Rotating $\mathbf{X}^{0,0}$ by $\alpha$ degrees around the $x$-axis, creating a tilted plane, and

2) Rotating $\mathbf{X}^{\alpha, 0}$ around the $z$-axis by $\beta$ degrees.

Figure 2 visualizes the effect of $\alpha$ and $\beta$. From this point, we consider $\mathbf{X}^{\alpha, \beta}$ to be the discrete reformation of the ultrasound volume onto the plane.

As each 3D volume is centered around the mitral valve, each plane $\mathbf{X}^{\alpha, \beta}$ - for reasonably small values of $\alpha-$ has two points of intersection between the mitral annulus and the plane. Given the mitral annulus label coordinates - introduced in Section II-A — we calculated the two intersections by linear interpolation. These two points are denoted $\mathbf{y}^{\alpha, \beta, l}$ and $\mathbf{y}^{\alpha, \beta, r}$, with $l$ and $r$ representing the left and right point in the generated image.

For a given $3 \mathrm{D}$ volume, we extracted a collection of images with corresponding annotations:

$$
\left\{\mathbf{X}^{\alpha, \beta}, \mathbf{y}^{\alpha, \beta, l}, \mathbf{y}^{\alpha, \beta, r}\right\} \text { for } \alpha \in \mathcal{S}_{\alpha}, \beta \in \mathcal{S}_{\beta},
$$


with $\mathcal{S}_{\alpha}=\left\{\alpha_{i}\right\}_{i=1}^{n_{\alpha}}$, a collection of discrete angles, and $n_{\beta}$ angles $\mathcal{S}_{\beta, n_{\beta}}$ evenly distributed angles between $0^{\circ}$ and $360^{\circ}$ a full rotation around the $z$-axis:

$$
\mathcal{S}_{\beta, n_{\beta}}=\left\{i \cdot 360 / n_{\beta}\right\}_{i=1}^{n_{\beta}} .
$$

The training, validation, and test data was generated by selecting appropriate $\mathcal{S}_{\alpha}$ and $\mathcal{S}_{\beta}$ and repeating this for all 3D volumes, as described in Section II-G.

\section{Fully convolutional network}

The first part of the model is a Convolutional Neural Network (CNN) without any fully connected layers, i.e., a fully convolutional network. We used the U-net [23] architecture, with the following remarks:

- The input dimension was $128 \times 128 \times 1$

- The output dimension was $128 \times 128 \times 2$ : one heatmap for each of the two points the model will predict, respectively denoted with $l$ and $r$ for the left and right point

- Padding was used for convolutions, keeping the input and output dimensions at the same size

- The following model parameters were varied as part of the hyperparameter search, see Section II-G:

- U-net depth, i.e., the number of max poolings

- Filters in the first layer - with each max pooling (depth) yielding twice as many filters - as in [23]

While U-net models are normally used in 2D segmentation, we apply the soft-argmax [24] to the output layers of the U-net, in order to do coordinate regression.

\section{Soft-argmax function}

In order to define a fully differentiable coordinate regression network - see Section II-E - we used the soft-argmax [24]. We follow the soft-argmax notation of [25].

Let $\hat{\mathbf{s}} \in \mathbb{R}^{n_{x} \times n_{y}}$ be a generic matrix variable. Given a single heatmap $\hat{\mathbf{s}}$, the softmax is:

$$
\Phi\left(\hat{\mathbf{s}}_{i, j}\right)=\frac{e^{\hat{\mathbf{s}}_{i, j}}}{\sum_{k=1}^{n_{x}} \sum_{l=1}^{n_{y}} e^{\hat{\mathbf{s}}_{k, l}}} .
$$

We define $\boldsymbol{\Phi}$ as the pixel-wise application of the softmax, $\Phi$ :

$$
\boldsymbol{\Phi}(\hat{\mathbf{s}}): \boldsymbol{\Phi}_{i, j}(\hat{\mathbf{s}})=\Phi\left(\hat{\mathbf{s}}_{i, j}\right)
$$

and denote the softmax normalization of $\hat{\mathbf{s}}$ by:

$$
\mathbf{s}=\boldsymbol{\Phi}(\hat{\mathbf{s}}),
$$

i.e., we denote the softmax normalization of a heatmap $\hat{s}$ by $\mathrm{s}$. We now apply soft-argmax function to $\mathbf{s}$ - calculating the mass center of the heatmap. Given a normalized heatmap, $\mathbf{s}$, the soft-argmax is defined by:

$$
\Psi^{d}(\mathbf{s})=\sum_{i=1}^{n_{x}} \sum_{j=1}^{n_{y}} \mathbf{W}_{i, j}^{d} \mathbf{s}_{i, j},
$$

for $d \in\{x, y\}$ where $\mathbf{W}_{i, j}^{d}$ are normalized ramps along the $x$ and $y$ directions:

$$
\mathbf{W}_{i, j}^{x}=\frac{i}{n_{x}}, \mathbf{W}_{i, j}^{y}=\frac{j}{n_{y}} .
$$

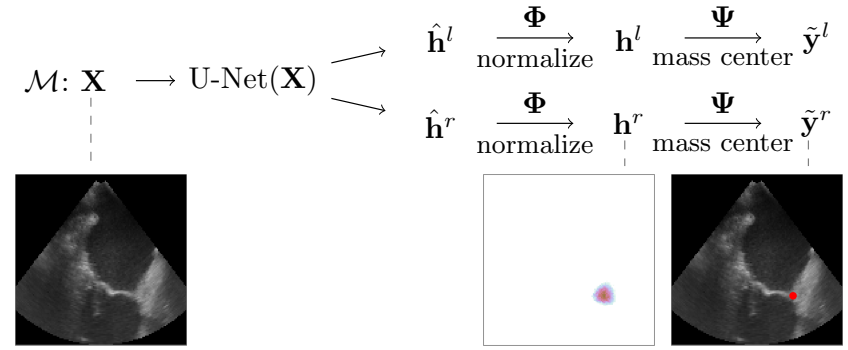

Fig. 3. Overview of CNN model with soft-argmax, as described in Section II-E. The CNN outputs two heatmaps that are normalized using softmax, $\boldsymbol{\Phi}$, see (5). The soft-argmax, $\boldsymbol{\Psi}$, see (7), is applied to the normalized heatmaps $\mathbf{h}^{l}$ and $\mathbf{h}^{r}$, to get the output coordinates $\tilde{\mathbf{y}}^{l}$ and $\tilde{\mathbf{y}}^{r}$, see (8).

The scalars $\Psi^{x}(\mathbf{s})$ and $\Psi^{y}(\mathbf{s})$ are the mass centers of $\mathbf{s}$ along the $x$ and $y$ axes, respectively. We define:

$$
\mathbf{\Psi}(\mathbf{s})=\left(\Psi^{x}(\mathbf{s}), \Psi^{y}(\mathbf{s})\right)^{T}
$$

to denote the mass center of $\mathbf{s}$. We take advantage of the differentiability of the soft-argmax — see, e.g., [25] — when defining our loss function in the following section.

\section{E. Model and loss function}

We define our model, $\mathcal{M}$, using the $\mathrm{CNN}$ described in Section II-C, combined with the soft-argmax defined in Section IID. The $\alpha$ and $\beta$ notation is omitted in this section to simplify notation. The CNN outputs two heatmaps, $\hat{\mathbf{h}}^{l}$ and $\hat{\mathbf{h}}^{r}$, for left and right points, respectively. We use $p$ to denote either $l$ or $r$, i.e., $\hat{\mathbf{h}}^{p}$ and $\mathbf{y}^{p}$.

We apply (6) to normalize $\hat{\mathbf{h}}^{p}$, i.e., $\mathbf{h}^{p}=\boldsymbol{\Phi}\left(\hat{\mathbf{h}}^{p}\right)$ and calculate the mass center of $\mathbf{h}^{p}$ using (7):

$$
\tilde{\mathbf{y}}^{\mathbf{p}}=\mathbf{\Psi}\left(\mathbf{h}^{p}\right) \text {. }
$$

We use the notation $\tilde{\mathbf{y}}^{\mathbf{p}}$ for the prediction estimate of $\mathbf{y}^{p}$. The flow chart in Figure 3 shows the steps of the complete model, $\mathcal{M}$. We consider the output of $\mathcal{M}(\mathbf{X})$ to be the normalized heatmaps and their mass centers, i.e., $\mathcal{M}(\mathbf{X})=$ $\left\{\mathbf{h}^{l}, \mathbf{h}^{r}, \tilde{\mathbf{y}}^{l}, \tilde{\mathbf{y}}^{r}\right\}$.

As previously noted, the soft-argmax is fully differentiable, allowing direct optimization of the CNN parameters of $\mathcal{M}$ with respect to label coordinates $\mathbf{y}^{l}$ and $\mathbf{y}^{r}$. We define the Euclidean loss between the true and predicted coordinates:

$$
\mathcal{L}_{\text {euc }}\left(\mathcal{M}(\mathbf{X}), \mathbf{y}^{p}\right)=\left\|\mathbf{y}^{p}-\tilde{\mathbf{y}}^{p}\right\|_{2},
$$

for $p \in\{l, r\}$.

As $\mathbf{h}^{p}$ are softmax normalized heatmaps, we can interpret them as probability distributions. Ideally, $\mathcal{M}(\mathbf{X})$ produces heatmaps $\mathbf{h}^{p}$ that closely resemble a normal distribution centered around the target coordinate $\mathbf{y}^{p}$, for $p \in\{l, r\}$. As proposed by Nibali et al. [26], we apply divergence-based loss to $\mathbf{h}^{p}$ to regularize the CNN towards this behavior. Specifically, we define:

$$
\mathcal{L}_{\text {reg }}\left(\mathcal{M}(\mathbf{X}), \mathbf{y}^{p}\right)=D\left(\mathbf{h}^{p} \| \mathcal{N}_{\mathbf{y}^{p}, \sigma_{D}}\right),
$$

where $D(\cdot \| \cdot)$ is the Jensen-Shannon divergence and $\mathcal{N}_{\mathbf{y}^{p}, \sigma_{D}}$ is a normal distribution with $\mu=\mathbf{y}^{p}$ and standard deviation $\sigma_{D}$ 


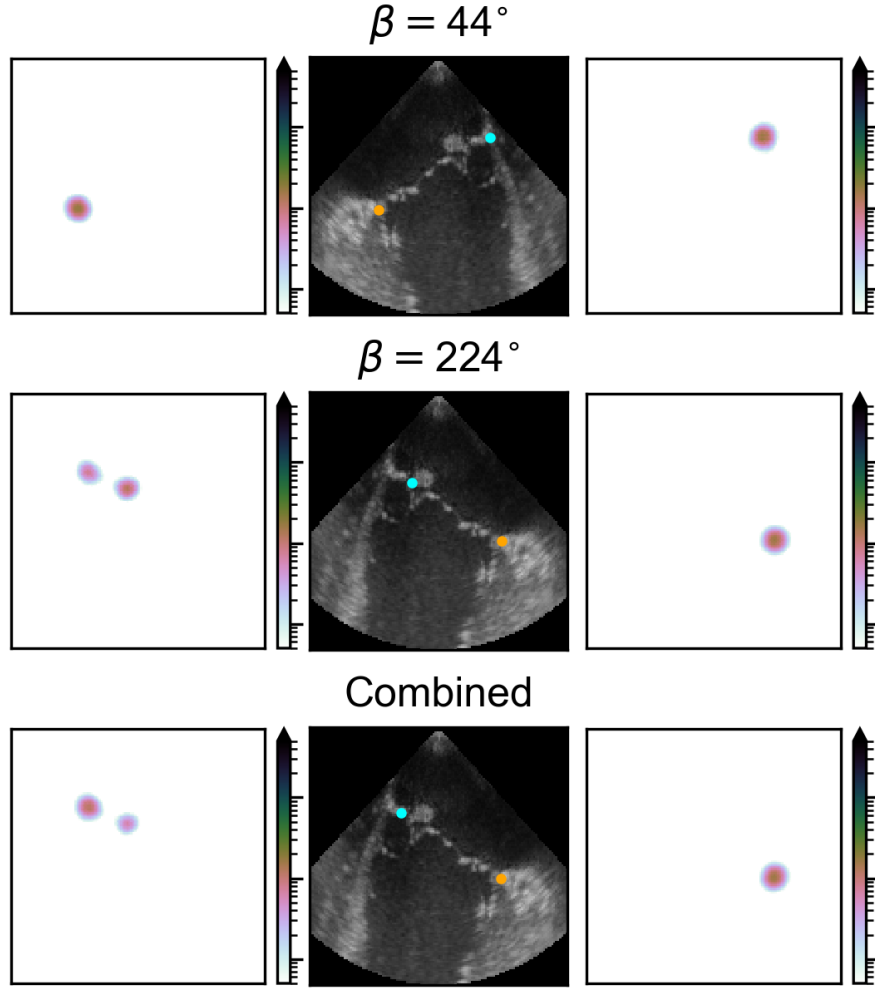

Fig. 4. Example output from the CNN for two planes from the same volume, $\mathbf{1 8 0}^{\circ}$ degrees apart. The left and right column respectively shows $\mathbf{h}^{l}$ and $\mathbf{h}^{r}$ from the image in the center column. The third row illustrates the application of (12) with the left image showing $\mathbf{h}^{c}$ at $\beta=224^{\circ}$ and the right showing the mirror image of $h^{c}$ at $\beta=44^{\circ}$. Orange point (left point in first row, right point in second and third row): The predictions are well aligned, resulting in one mode in the combined heatmap. Cyan point (right point in first row, left point in second and third row): While the heatmap at $\beta=44^{\circ}$ has a single mode, the heatmap at $\boldsymbol{\beta}=\mathbf{2 2 4}^{\circ}$ has detected two positions, resulting in two modes, cf. Section II-F. The combined heatmap, using (12), still has two modes before the continuity prior is applied. The colormap use a logarithmic scale from $5 \mathrm{e}-4$ to $5 \mathrm{e}-1$.

Our complete loss function used for training the network is the weighted sum of the Euclidean loss and the regularization loss:

$$
\begin{array}{r}
\mathcal{L}\left(\mathcal{M}(\mathbf{X}), \mathbf{y}^{l}, \mathbf{y}^{r}\right)=\sum_{p \in\{l, r\}}\left[\mathcal{L}_{\text {euc }}\left(\mathcal{M}(\mathbf{X}), \mathbf{y}^{p}\right)+\right. \\
\left.\lambda \mathcal{L}_{\text {reg }}\left(\mathcal{M}(\mathbf{X}), \mathbf{y}^{p}\right)\right]
\end{array}
$$

with the hyperparameter $\lambda$ balancing the contribution between $\mathcal{L}_{\text {euc }}$ and $\mathcal{L}_{\text {reg. }}$.

Note that the model $\mathcal{M}$ operates on individual 2D images. In the following section we introduce the final step of our method: a simple continuity prior that exploits the spatial continuity of the mitral annulus - iteratively building the 3D curve segmentation.

\section{F. A continuity prior}

Consider a model, $\mathcal{M}$, optimized with respect to the loss function defined in Section II-E. Most heatmaps generated using $\mathcal{M}$ contains a single mode, as illustrated in the first row of Figure 4. The network may detect multiple candidate regions - as illustrated in the left heatmap on the second row of Figure 4. In the following, a continuity prior is proposed for suppressing such modes.

Set $\alpha=0$ and consider the planes $\mathbf{X}^{0, \beta}, \beta \in \mathcal{S}_{\beta}$ from a single $3 \mathrm{D}$ volume. For any $\beta, \mathbf{X}^{0, \beta}$ and $\mathbf{X}^{0, \beta+180^{\circ}}$ are mirror images, as illustrated in Figure 4 . We take advantage of this by averaging $\mathbf{h}^{0, \beta_{i}, l}$ and the mirror reflection of $\mathbf{h}^{0, \beta_{i}+180^{\circ}, r}$, i.e.:

$$
\mathbf{h}^{0, \beta_{i}, \text { combined }}=\frac{1}{2}\left(\mathbf{h}^{0, \beta_{i}, l}+\operatorname{Ref}\left(\mathbf{h}^{0, \beta_{i+1}+180^{\circ}, r}\right)\right),
$$

with $\operatorname{Ref}(\cdot)$ denoting the reflection transform. The shorthand notation $c$ refers to combined in what follows. The third row of Figure 4 illustrates the effect of (12).

The proposed method takes advantage of the continuity of the mitral annulus. Using the planes introduced in (3) and (4), we know that $\mathbf{X}^{0, \beta_{i}}$ and $\mathbf{X}^{0, \beta_{i+1}}$ differ only by a small rotation around the $z$-axis, given that the number of rotations, $n_{\beta}$, is sufficiently high. Since the mitral annulus is a continuous structure, we have:

$$
\mathbf{y}^{0, \beta_{i}, p} \approx \mathbf{y}^{0, \beta_{i+1}, p}
$$

Therefore, predictions of consecutive planes should also fulfill this property:

$$
\tilde{\mathbf{y}}^{0, \beta_{i}, p} \approx \tilde{\mathbf{y}}^{0, \beta_{i+1}, p}
$$

We applied windowing of the prediction heatmaps iteratively to the rotational planes in order to suppress modes in the heatmaps that are incompatible with the continuity constraint.

The angular momentum $I(\cdot)$, defined below, depends quadratically on distance from the center of mass. The angular momentum is:

$$
I(\mathbf{h})=\sum_{i, j} d_{i, j}^{2} \mathbf{h}_{i, j},
$$

where $d_{i, j}$ is the distance to the mass center of $\mathbf{h}$. A low angular momentum $I\left(\mathbf{h}^{0, \beta, c}\right)$ indicates that the two heatmaps (12) coincide and $I\left(\mathbf{h}^{0, \beta, c}\right)$ has a single mode, e.g., as illustrated with the orange point in Figure 4. The combined heatmap corresponding to the cyan point has two modes, and as such a higher angular mass.

The low spread can be a proxy for high confidence. Set the initial plane $\mathbf{X}^{0, \beta_{i}}$ to the plane with the lowest angular momentum:

$$
\beta_{i_{0}}=\underset{\beta \in \mathcal{S}_{\beta}}{\arg \min } I\left(\mathbf{h}^{0, \beta, c}\right) .
$$

Define the windowing of $\mathbf{h}$ as the element wise product of $\mathbf{h}$ and $\mathbf{w}_{w}, \sigma_{w}^{2}$ - a discretized Gaussian kernel with standard deviation $\sigma_{w}$, centered at $\boldsymbol{\mu}_{w}$ :

$$
\mathcal{W}\left(\mathbf{h}, \boldsymbol{\mu}_{w}\right)=\mathbf{h} \circ \mathbf{w}_{\boldsymbol{\mu}_{w}, \sigma_{w}} .
$$

The iterative algorithm is outlined in Algorithm 1. Given the heatmaps, $\left\{\mathbf{h}^{0, \beta, c}\right\}_{\beta \in \mathcal{S}_{\beta}}$, from a single volume, Algorithm 1 yields the final predictions for each $2 \mathrm{D}$ plane, $\left\{\mathbf{y}_{\text {final }}^{\beta}\right\}_{\beta \in \mathcal{S}_{\beta}}$. Figure 5 visualizes the effect of the continuity prior.

The predicted 3D geometry of the mitral annulus yields from projecting $\left\{\mathbf{y}_{\text {final }}^{\beta}\right\}_{\beta \in \mathcal{S}_{\beta}}$, to $3 \mathrm{D}$ space. Figure 6 gives an overview of the proposed method. 
TABLE I

TRAINING, VALIDATION, AND TEST SPLIT

\begin{tabular}{|l|l|l|l|}
\hline Dataset & $\begin{array}{l}\text { Exams } \\
\text { (DICOM files) }\end{array}$ & $\begin{array}{l}\text { Volumes } \\
\text { 3D frames }\end{array}$ & $\begin{array}{l}\text { Images } \\
\text { 2D png files }\end{array}$ \\
\hline Training & $55(74)$ & 459 & 195,250 \\
Validation & $15(18)$ & 106 & 9,450 \\
Test & $19(19)$ & 135 & 12,150 \\
\hline Total & $89(111)$ & 700 & 216,850 \\
\hline
\end{tabular}

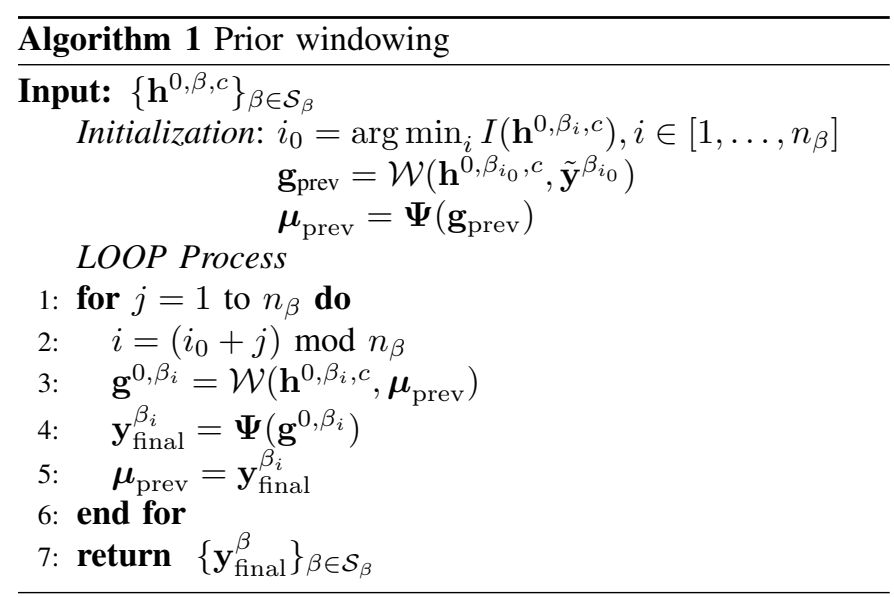

\section{G. Experiments}

The examinations were randomly split into the training, validation, and test sets, ensuring that DICOM files from the same examinations were grouped together - in order to avoid bias across the three datasets. An overview of the data split is given in Table I, including the number of examinations, the number of DICOM files, and the total number of 3D volumes for each of the three datasets. Note that the examinations in the test set only contains one DICOM file each, however, each of the DICOM files contains multiple frames.

We generated the $2 \mathrm{D}$ images from the $3 \mathrm{D}$ volumes as described in Section II-B, with the choice of $\mathcal{S}_{\alpha}$ and $\mathcal{S}_{\beta}$ summarized in Table II:

- Training set: To maximize the utilization of each training set volume, planes were extracted with several tilt angles, $\alpha$.

A thresholding between $\mathbf{y}^{\alpha, \beta, l}$ and $\mathbf{y}^{\alpha, \beta, r}$ was applied to ensure that the tilted planes in the training set pass through the mitral valve. The threshold was set to 2.2 $\mathrm{cm}$ - the $5 \%$ percentile mitral annulus diameter for adult females, according to the results given in [27].

- Validation set $\&$ test set: A single rotation around the $z$-axis was extracted for each 3D volume in the validation set and the test set. As discussed in Section II$\mathrm{F}, \mathcal{S}_{\alpha}=\{0\}$ was used in order to exploit the $180^{\circ}$ mirror symmetry of (12).

For all three sets $n_{\beta}=90$ was used, i.e., one plane every $4^{\circ}$ rotation around the $z$-axis is extracted. The pixel values in each plane were normalized to $[0,1]$. The total number of $2 \mathrm{D}$ planes is given in Table I.

Based on preliminary experiments, model training was carried out with a grid search over the following hyperparameters:

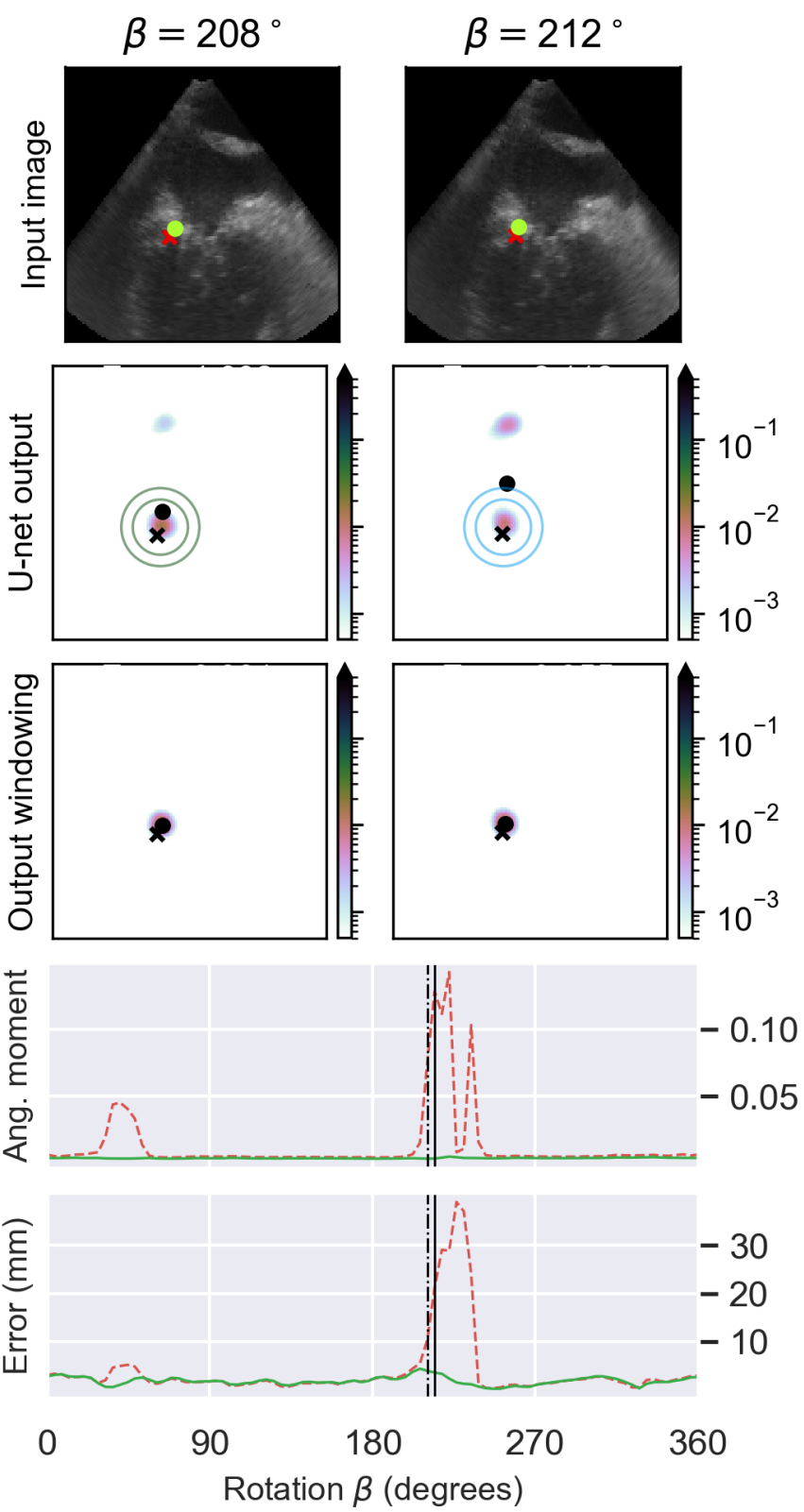

Fig. 5. Illustration of the effect of windowing on two consecutive 2D planes from a single 3D volume, as described in Algorithm 1. First row: Input image with label (red cross) and final prediction (green circle). Second row: Prediction made by U-net. In these particular images, two candidate locations are identified by the U-net. As a result, the raw prediction (black circle) is located between the two modes. This is also reflected in the plot with the angular momentum. The contour lines show the 0.1 and 0.01 levels of the Gaussian kernel, used for windowing (13). Label is shown as the black cross. Third row: Final heatmap, after applying windowing. The black circle shows the final prediction in the plane. Label is shown as the black cross. Fourth row: Angular moment, $I(\cdot)$ before (red, dotted line) and after (green, solid line) windowing. The black lines in row four and five highlight the angles of the two planes shown in the figure: $\beta=208^{\circ}$ and $\beta=\mathbf{2 1 2}^{\circ}$. Fifth row: Prediction error before (red, dotted line) and after (green, solid line) windowing. Note that the windowing removes the second mode of the heatmap, significantly reducing the prediction error.

- U-net depth: the number of max-pool layers in U-net model, as described in Section II-C

- no. filters: number of filters in initial U-net layer, as 


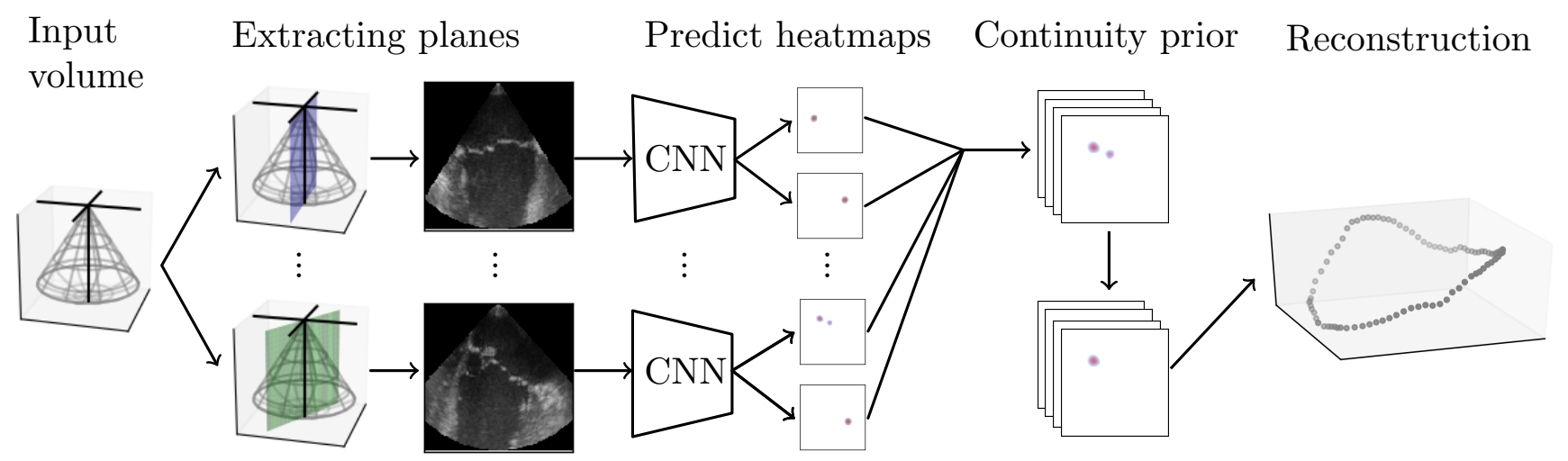

Fig. 6. Overview of the proposed method. The input volume (Section II-A) is being processed by extracting 2D planes (Section II-B) centered around the centerline. Using a trained 2D CNN (Sections II-C, II-D, II-E) heatmaps for the mitral annuls points are predicted. The 3D geometry of the mitral annulus is reconstructed by sequentially applying a continuity prior (Section II-F).

TABLE II

Planes generated PER 3D volume

\begin{tabular}{|l|l|l|l|}
\hline Dataset & $\mathcal{S}_{\alpha}$ & $\mathcal{S}_{\beta, n_{\beta}}$ & Threshold $^{\text {a }}$ \\
\hline Training & $\{0,2,4,6,8\}$ & $n_{\beta}=90$ & $2.2 \mathrm{~cm}$ \\
Validation & $\{0\}$ & $n_{\beta}=90$ & $2.2 \mathrm{~cm}$ \\
Test & $\{0\}$ & $n_{\beta}=90$ & NA \\
\hline
\end{tabular}

${ }^{a}$ Only keeping images where the distance between the two mitral annulus intersections is larger than this value, as described in Section II-G.

described in Section II-C

- $\lambda$ : weight balancing the contribution between the Euclidean loss and the divergence loss, as defined in (11)

- $\sigma_{\mathcal{D}}$ : variance of normal distribution $\mathcal{N}_{\mathbf{y}, \sigma_{D}}$ used in the divergence loss (10)

- $\nu$ : learning rate used in the model optimization

The hyperparameter search space is given in Table III, where the values were selected based on the preliminary experiments. We trained one model for each Cartesian product of hyperparameters given in the table.

Each model was initialized with He initialization [28] and used the Adam optimizer [29], with parameters $\beta_{1}=0.9, \beta_{2}=$ 0.999 . We applied exponential decay of the learning rate, with decay parameter $\gamma=0.95$, applied between each epoch. All models were trained with a batch size of 32 and an epoch size of 9024 , where images were randomly sampled using a weight proportional to the number of png images generated from the same examination. Preliminary experiments showed that models converged quickly. Due to this, we applied early stopping — with a maximum of 10 epochs — in order to avoid overfitting.

We selected the model with the overall lowest mean validation error as the final model, $\mathcal{M}_{\text {final }}$. The windowing size, $\sigma_{w}$, was subsequently tuned by applying the continuity prior on the validation set with the values $\sigma_{w} \in\{3, \ldots, 10\}$ - using the final model $\mathcal{M}_{\text {final }}$.

The error on the test set was calculated by running inference a single time on the selected model, $\mathcal{M}_{\text {final }}$, and applying Algorithm (1), with the selected $\sigma_{w}$.

We implemented our method in Python 3.6.2, using PyTorch

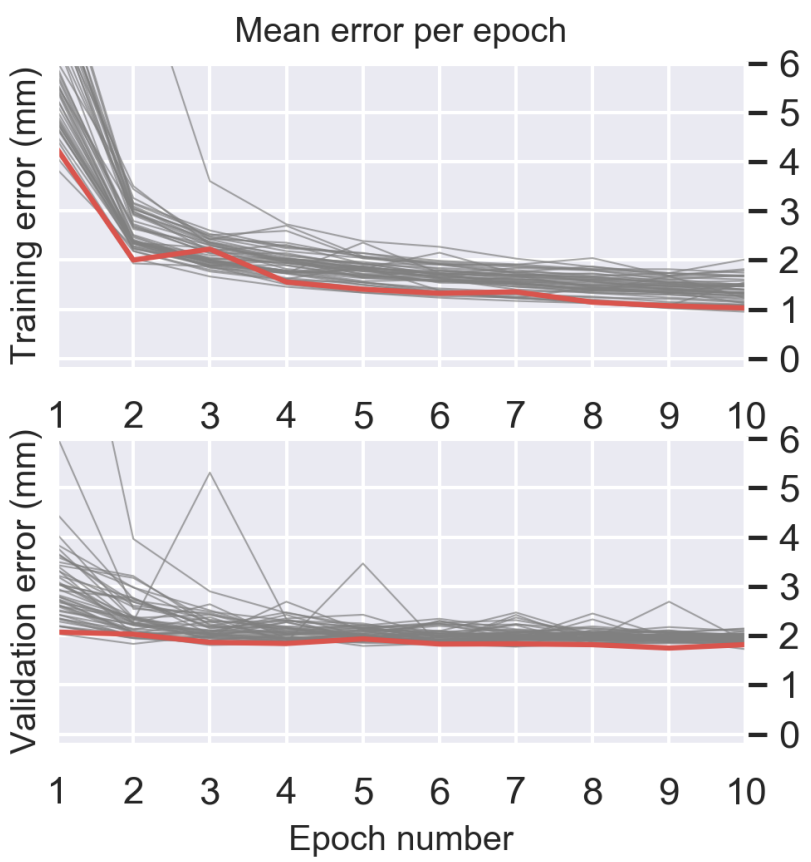

Fig. 7. Mean error per epoch for the training and validation set. Each gray line represents one model training. The orange line shows the learning curves of the final model. Note that the learning curve of five models that diverged are not shown.

1.0. The PIP package dsntnn [26] was used for the softargmax and the U-net implementation was based on the implementation at https://github.com/jaxony/unet-pytorch. All models were trained on a machine with an Intel Xeon CPU E5-2620, using one Nvidia Quadro P6000 GPU.

\section{H. Clinical parameters}

The area and perimeter of the mitral annulus are calculated and reported for the mid-systolic frame of each examination. The perimeter is calculated as the Euclidean distance across the points on the annulus. The area is estimated as the sum of consecutive triangles sharing a fixed reference vertex. Since the aortic outlet is a challenging anatomical area, the directly opposite point was selected as the reference vertex. 
TABLE III

HYPERPARAMETER SEARCH

\begin{tabular}{l|l|l|l|l|c|}
\hline & $\begin{array}{l}\text { U-net } \\
\text { depth }\end{array}$ & $\begin{array}{l}\text { no. } \\
\text { filters }\end{array}$ & $\lambda$ & $\begin{array}{l}\sigma_{\mathcal{D}} \\
\text { (pixel) }\end{array}$ & $\nu$ \\
\hline Exp.\#1 & 3,4 & 32,64 & 1,5 & $1,3,5$ & $1 \mathrm{e}-3,5 \mathrm{e}-4,1 \mathrm{e}-4$ \\
Exp.\#2 & 5 & 32,64 & 1,5 & 3,5 & $5 \mathrm{e}-4,1 \mathrm{e}-4$ \\
\hline
\end{tabular}
Overview of hyperparameter search. As described in Section II-G,
we trained one model for each Cartesian product of hyperparame-
ters.

TABLE IV

RESULTS OF THE FINAL MODEL

\begin{tabular}{|c|c|c|}
\hline & Weighted mean error $(\mathrm{mm})$ & Standard deviation $(\mathrm{mm})$ \\
\hline validation set & 1.9 & 1.7 \\
test set & 2.0 & 1.9 \\
\hline
\end{tabular}

\section{Results}

In the hyperparameter experiment, 88 model configurations were evaluated, of which the majority converged to a mean validation error in individual planes between $1.7 \mathrm{~mm}$ and 2.1 $\mathrm{mm}$, as illustrated in Figure 7. The selected model — based on the validation loss $-\mathcal{M}_{\text {final }}$, was trained with the following hyperparameters:

- U-net depth: 5

- Starting filters: 32

- Learning rate: $\nu=5 \mathrm{e}-4$

- $\lambda=5$

- $\sigma_{\mathcal{D}}=3$ (pixels).

The results with $\sigma_{w}$ between 6 and 10 performed the same. The value $\sigma_{w}=6$ (pixels) was selected.

We report the weighted in-plane prediction error and standard deviation across all 2D planes for the selected model. The weighting, see Appendix I, takes into account that the examinations have a different number of volumes. The final weighted mean error was $2.0 \mathrm{~mm}$, with weighted standard deviation of $1.9 \mathrm{~mm}$, see Table IV.

The errors for each examination in the test set are presented as a box plot in Figure 8, with average error for each examination is indicated with green triangles. Figure 9 shows the error distribution of the $\sim 12.000$ planes in the test set.

The area and perimeter of the mitral annulus predictions described in Section II-H - are reported in Table V.

\section{DISCUSSION}

We have presented a fully automatic method for mitral annulus segmentation in 3D echocardiography volumes. The mean prediction error of our method on the test set (on 135 3D volumes, cf. Table I) is comparable with both [16] and [18], as these works also report the average root mean square error in 2D planes. Schneider et al. [16] reported an error of $1.8 \pm 0.8 \mathrm{~mm}$ across a dataset of 10 volumes - measured

TABLE V

Clinical parameters RESULtS OF THE FINAL MODEL

\begin{tabular}{|c|c|c|}
\hline & Relative error & Absolute error \\
\hline Perimeter & $6.1 \pm 4.5 \%$ & $8.1 \pm 6.0 \mathrm{~mm}$ \\
Area & $11.94 \pm 10.0 \%$ & $1.6 \pm 1.4 \mathrm{~cm}^{2}$ \\
\hline
\end{tabular}

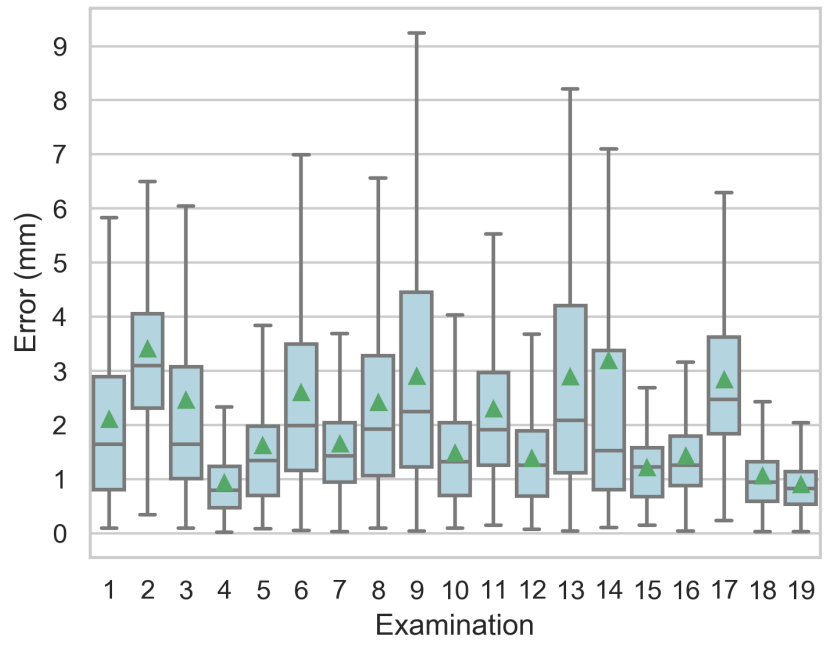

Fig. 8. Box plot shows the distribution of errors across the 19 patients in the test set. The mean error for each sample is indicated with the green triangles. The box plot indicates the median error and the quartiles, and the whiskers show the $\mathbf{1 . 5}$ times the interquartile range. Outliers are not shown in the plot.

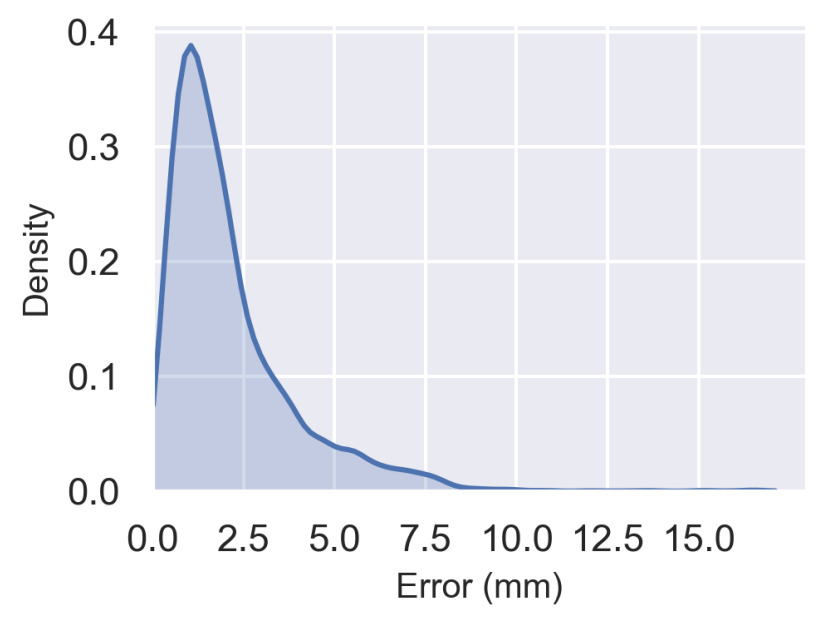

Fig. 9. Distribution of prediction errors for the $\mathbf{1 2 . 1 5 0}$ individual images in the test set.

against the mean coordinates of labels made by ten trained experts. Tiwari et al. [18] reported an average localization error of $2.6 \mathrm{~mm}$, using data from 15 patients.

Both [16] and [18] require one manual input point. Schneider et al. used a surface curvature energy term for encouraging smooth local changes, while Tiwari et al. utilizes a modelbased prior with a naive Bayes classifier. Lastly, Ionasec et al. introduced a fully automatic method [12] using a physiological model, but the reported results are not directly comparable to our method. Our proposed method requires no manual input and has the continuity of the mitral annulus as its only prior.

One of the limitations of our study is that only systolic frames are included. Ionasec et al. [12] and Schneider et al. [17] both published methods covering the full cardiac cycle. The reason for not including diastolic frames in this work is the lack of annotations. 4D AutoMVQ was designed to study mitral insufficiency, a systolic condition, and thus only covers 
the systolic phase. Our proposed method would likely also work for the diastole - given sufficient training data.

In general, our experiments would benefit from additional data. The literature suggests that inter-observer variability is significant [16]. The impact of this variability could be investigated using consensus reading of multiple experts to improve the quality of the annotations. This additional data could be used both for training and validation, and also for exploring alternative approaches.

While our data is volumetric, we chose to take advantage of the symmetry of the left ventricle in our approach. Extracting $2 \mathrm{D}$ planes from the $3 \mathrm{D}$ volumes allowed us to generate a higher number of training samples - by varying $\alpha$ and $\beta$ - than if we had applied a 3D method directly. While the correlation between neighboring planes in the volume is high, our approach can be seen as a natural augmentation of the data - since near planes contain different noise and slight differences in the structure. Training the model on planes offset to the depth direction $(\alpha \neq 0)$ likely increases the robustness to natural variance in the orientation of the mitral annulus and to non-centered acquisitions.

The continuity prior proposed in our work takes further advantage of symmetry - improving the stability of the model. We found that the average test error is not reduced significantly by applying the continuity prior, however, predictions with multiple modes are improved, reducing outlier predictions.

We performed a limited hyperparameter search to ensure that the method was stable with respect to hyperparameter change. While a more extensive search could generate better models, we aimed to avoid overfitting the model to the training set. The validation curves of Figure 7 indicates that the method quickly converges to a plateau with an average error of approximately $2 \mathrm{~mm}$, and that the method is quite robust to the selected hyperparameters.

To understand the contribution of the loss components, a small subsequent experiment was conducted on the validation set. The experiment indicated that the regularization loss is required for convergence, and that the model can converge using the regularization loss alone. The soft-argmax would still be important in the proposed method, as it is used to calculate the prediction coordinates and the continuity prior. Future work could investigate the contribution of the different losses further.

The errors on the test set of our final model are concentrated in the lower end of the error range, as can be seen in Figure 9. Some outliers are discussed below. Figure 10 shows six representative predictions. The difference between the prediction and the label in these examples would likely be in the range of inter-observer variability. In particular, the inter-observer variance between the ten experts providing annotations in [16] is in the range of $1-2 \mathrm{~mm}$. Note that the prediction error in the right column, second row $(2.5 \mathrm{~mm})$ of Figure 10 is higher than our average error.

Figure 11 shows the worst predictions for examinations 9, 6,3 , and 14 . The plane from examination 9 does not include the ventricular wall - which could be the cause of confusion for the model. Both planes from examination 6 and 3 show the aortic outflow region. This region can be hard to annotate
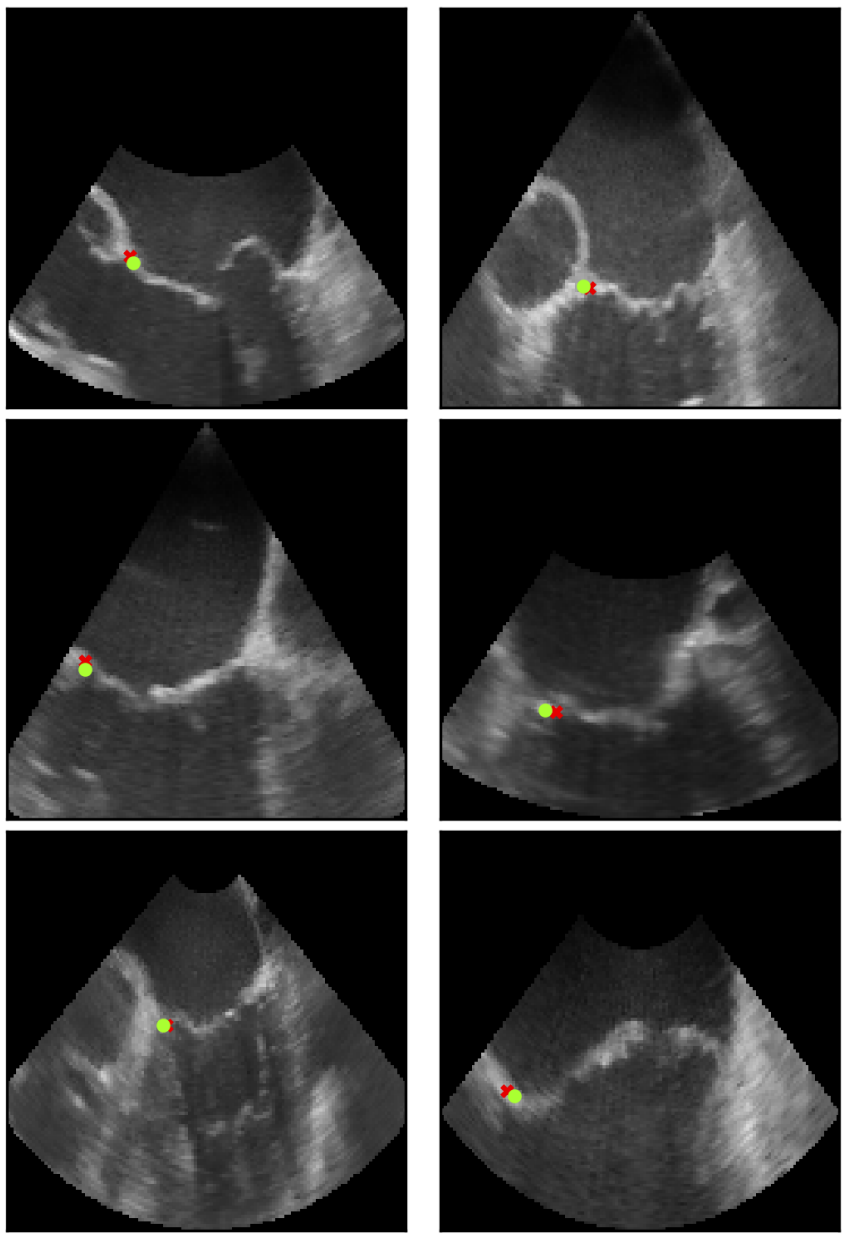

Fig. 10. Representative predictions made by our method. The mean error of the images in this figure is less than $1.7 \mathrm{~mm}$. The right image in the second row has a prediction error of $\mathbf{2 . 5} \mathbf{~ m m}$. Labels are marked with a red cross and predictions with a green circle.

without spatial and temporal context - in particular in the systole where the aortic valve is mostly open. Finally, the image from examination 14 shows the highest prediction error in the entire test set. While the median error for examination 14 is $1.5 \mathrm{~mm}$ (see Figure 8), the model fails in a sector of the volume - as shown in the bottom plot of Figure 12 .

While the average prediction error in the planes is low, outlier predictions occur - as highlighted in Figure 11. Such outliers can lead to significant errors in global measurements, as the area and perimeter reported in Section III, and would require manual correction. Figure 12 visualize the results of our method on two of the 3D volumes. The top plot depicts a volume from examination 4 , where the model performs well, while the bottom plot comes from examination 14 where the model — as mentioned above - fails in a sector of the volume. Introducing global context for the model, using a global prior or a direct 3D method, could reduce both pointwise and clinical measurement errors.

There are trade-offs between using a 2D or 3D model. A direct 3D method could potentially utilize more anatomical information. However, this would require more computational power, which would make it less suitable for implementation 

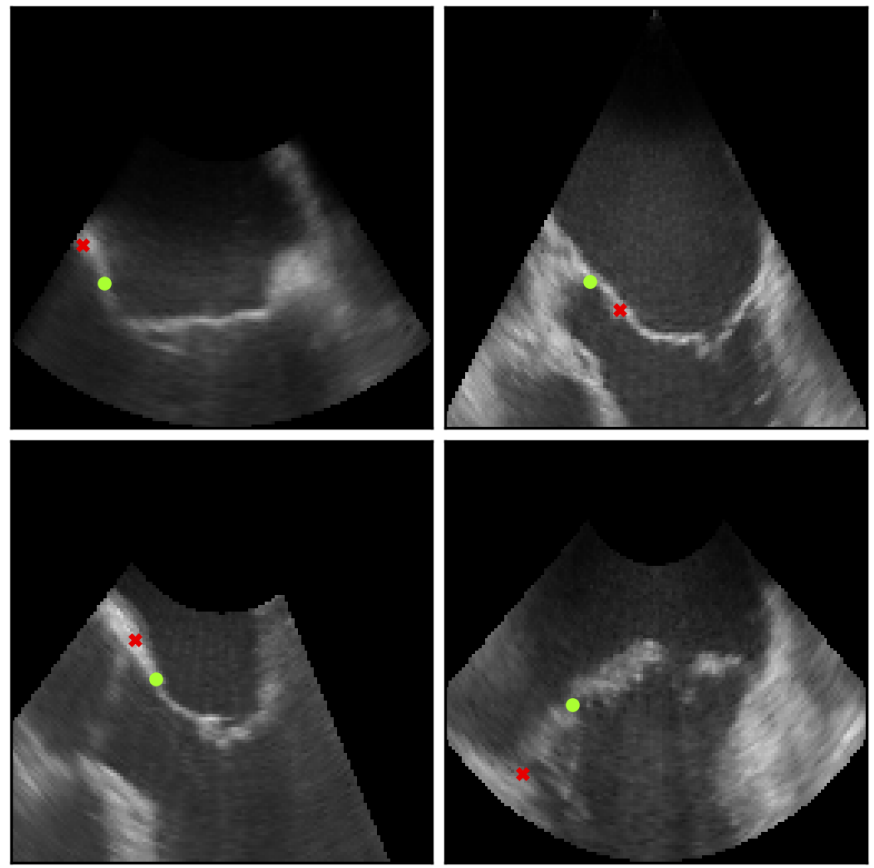

Fig. 11. The single worst prediction from the following four examinations - with the same numbering as in the box plot of Figure 8. First row, left to right: examinations 9 and 6 . Second row, left to right: examinations 3 and 14 . The right image on the second row is the overall worst prediction on our test set. Labels are marked with a red cross and predictions with a green circle.

in a scanner. By using a 2D CNN iteratively, the proposed method is efficient in terms of both runtime and memory requirements - with an inference time of $1.71 \pm 0.06 \mathrm{sec}-$ onds per volume (across 100 runs on a machine using one Nvidia Quadro P6000 GPU and an Intel Xeon CPU E5-2620). Faster inference and reduction of computational cost could be achieved by reducing the number of planes, $n_{\beta}$, in the inference. Changing $n_{\beta}$ would likely result in a different $\sigma_{w}$, due to the different distance between planes.

Adding a more advanced anatomical prior to the proposed method, and exploring alternative approaches with 3D CNNs are planned as future work. Alternatively, consistency across planes could be enforced in the network architecture, as demonstrated by Zheng et al. in [30] who used a 2D CNN sequentially - feeding predictions from the previous plane to a separate encoding contextual branch of the model. Another possible extension of the method could be the prediction of additional anatomical structures such as the mitral leaflet coaptation or the aortic valve. Future work could explore multi-task learning [31], [32] in order to also optimize for clinical parameters and additional structures during training.

\section{CONCLUSION}

We proposed a method for automatic detection of the mitral annulus within 3D TEE. The method is fully automatic and fast, based on convolutional neural networks, using no prior model. With a detection error of $2 \mathrm{~mm}$, it has the potential to automatically detect the position and orientation of the mitral valve in $3 \mathrm{D}$ images and to provide relevant clinical parameters
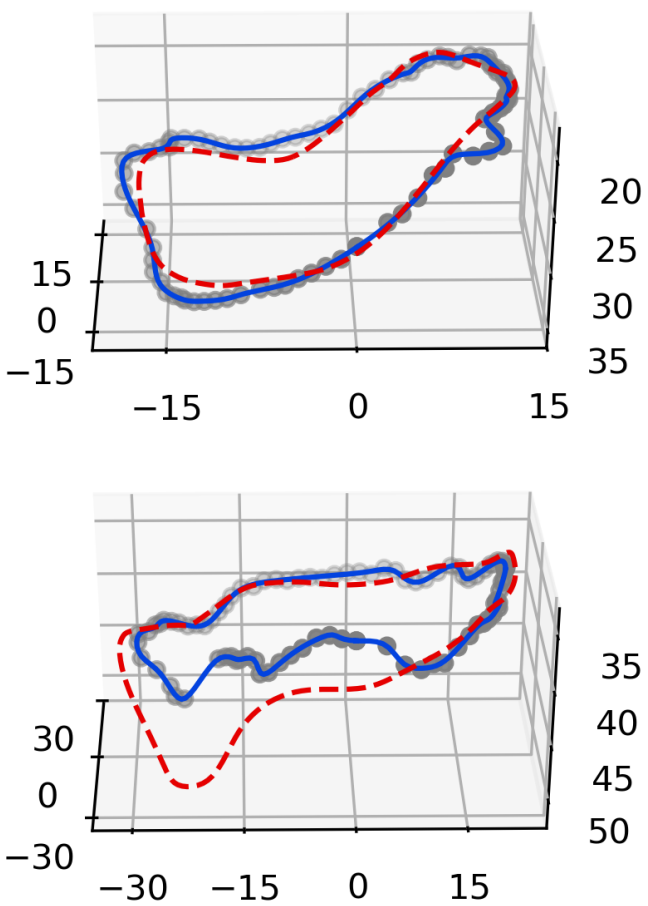

Fig. 12. Example predictions of mitral annulus valves projected in 3D. The grey circles show predictions from the method and the blue line shows a spline fitted to the points. The red (dashed) line show the target labels. The unit of the axes is $\mathrm{mm}$, with the probe at the origin. Top: Examination 4 - average error: $1.1 \mathrm{~mm}$. Bottom: Examination $14-$ average error: $3.3 \mathrm{~mm}$. As discussed in Section IV, the model fails in a region of examination 14 .

of the mitral apparatus. Results can also be used for diagnostics, patient selection, and planning of therapy in an area where the number of therapeutic options is rapidly increasing. Further, the tool could be used during cardiac interventions as guidance during mitral valve repair or replacement.

APPENDIX I

WEIGHTING FORMULA

Let the total number of examinations be denoted by $N_{\mathrm{e}}$ and the number of planes in examination $i$ - across all time frames and rotations - be denoted by $n_{i}$. Further, let $w_{i}=1 /\left(N_{\mathrm{e}} \cdot n_{i}\right)$ be a weighting term for each examination and finally let $E_{i, j}$ be the Euclidean error of the $j$-th plane in examination $i$. The weighted average error and weighted sample variance is then given by: 


$$
\begin{aligned}
\mu_{\mathrm{W}} & =\sum_{i=1}^{N_{\mathrm{e}}} w_{i} \sum_{j=1}^{n_{j}} E_{i, j} \\
& =\frac{1}{N_{\mathrm{e}}} \sum_{i=1}^{N_{\mathrm{e}}} \frac{1}{n_{i}} \sum_{j=1}^{n_{i}} E_{i, j}, \\
\sigma_{\mathrm{W}}^{2} & =\sum_{i=1}^{N_{\mathrm{e}}} w_{i} \sum_{j=1}^{n_{i}}\left(E_{i, j}-\mu_{\mathrm{W}}\right)^{2} \\
& =\frac{1}{N_{\mathrm{e}}} \sum_{i=1}^{N_{\mathrm{e}}} \frac{1}{n_{i}} \sum_{j=1}^{n_{i}}\left(E_{i, j}-\mu_{\mathrm{W}}\right)^{2} .
\end{aligned}
$$

\section{ACKNOWLEDGMENT}

The authors thanks David Völgyes for contributions through discussions, ideas, and concrete material for the paper; Andreas Austeng and Fritz Albregtsen for discussions and feedback on the method; and Kristin McLeod for data preparation.

\section{REFERENCES}

[1] P. A. Iaizzo, ed., Handbook of Cardiac Anatomy, Physiology, and Devices. New York, NY: Humana Press, 2nd ed. ed., 2009.

[2] M. Enriquez-Sarano, C. W. Akins, and A. Vahanian, "Mitral regurgitation," The Lancet, vol. 373, pp. 1382-1394, 42009.

[3] T. Gemechu, H. Mahmoud, E. H. Parry, D. I. Phillips, and M. H. Yacoub, "Community-based prevalence study of rheumatic heart disease in rural Ethiopia," European Journal of Preventive Cardiology, vol. 24, pp. 717 723,52017

[4] V. T. Nkomo, J. M. Gardin, T. N. Skelton, J. S. Gottdiener, C. G. Scott, and M. Enriquez-Sarano, "Burden of valvular heart diseases: a population-based study.," Lancet (London, England), vol. 368, pp. 10051011, 92006.

[5] R. A. Nishimura, A. Vahanian, M. F. Eleid, and M. J. Mack, "Mitral valve disease-current management and future challenges.," Lancet (London, England), vol. 387, pp. 1324-1334, 32016.

[6] L. Q. Rong, "An update on intraoperative three-dimensional transesophageal echocardiography," Journal of Thoracic Disease, vol. 9, pp. S271-S282, 42017.

[7] M. Pepi, G. Tamborini, A. Maltagliati, C. A. Galli, E. Sisillo, L. Salvi, M. Naliato, M. Porqueddu, A. Parolari, M. Zanobini, and F. Alamanni, "Head-to-Head Comparison of Two- and Three-Dimensional Transthoracic and Transesophageal Echocardiography in the Localization of Mitral Valve Prolapse," Journal of the American College of Cardiology, vol. 48, pp. 2524-2530, 122006.

[8] S. Ben Zekry, S. F. Nagueh, S. H. Little, M. A. Quinones, M. L. McCulloch, S. Karanbir, E. L. Herrera, G. M. Lawrie, and W. A. Zoghbi, "Comparative Accuracy of Two- and Three-Dimensional Transthoracic and Transesophageal Echocardiography in Identifying Mitral Valve Pathology in Patients Undergoing Mitral Valve Repair: Initial Observations," Journal of the American Society of Echocardiography, vol. 24, pp. 1079-1085, 102011.

[9] M. Izumo, M. Shiota, S. Kar, S. V. Gurudevan, K. Tolstrup, R. J. Siegel, and T. Shiota, "Comparison of Real-Time Three-Dimensional Transesophageal Echocardiography to Two-Dimensional Transesophageal Echocardiography for Quantification of Mitral Valve Prolapse in Patients With Severe Mitral Regurgitation," The American Journal of Cardiology, vol. 111, pp. 588-594, 22013.

[10] M.-S. Lee and T. Z. Naqvi, "A Practical Guide to the Use of Echocardiography in Assisting Structural Heart Disease Interventions," Cardiology Clinics, vol. 31, pp. 441-454, 82013.

[11] M. Garbi and M. J. Monaghan, "Quantitative mitral valve anatomy and pathology," Echo Research and Practice, vol. 2, p. R63, 92015.

[12] R. I. Ionasec, I. Voigt, B. Georgescu, Y. Wang, H. Houle, F. VegaHiguera, N. Navab, and D. Comaniciu, "Patient-specific modeling and quantification of the aortic and mitral valves from 4-D cardiac CT and TEE.," IEEE transactions on medical imaging, vol. 29, pp. 1636-51, 9 2010 .
[13] I. Aquila, A. González, C. Fernández-Golfín, L. M. Rincón, E. Casas, A. García, R. Hinojar, J. J. Jiménez-Nacher, and J. L. Zamorano, "Reproducibility of a novel echocardiographic 3D automated software for the assessment of mitral valve anatomy," Cardiovascular Ultrasound, vol. 14, p. 17, 122015

[14] J. Jeganathan, Z. Knio, Y. Amador, T. Hai, A. Khamooshian, R. Matyal, K. R. Khabbaz, and F. Mahmood, "Artificial intelligence in mitral valve analysis.," Annals of cardiac anaesthesia, vol. 20, pp. 129-134, 42017.

[15] S. Gandhi, W. Mosleh, J. Shen, and C.-M. Chow, "Automation, machine learning, and artificial intelligence in echocardiography: A brave new world," Echocardiography, vol. 35, no. 9, pp. 1402-1418, 2018.

[16] R. J. Schneider, D. P. Perrin, N. V. Vasilyev, G. R. Marx, P. J. del Nido, and R. D. Howe, "Mitral Annulus Segmentation From 3D Ultrasound Using Graph Cuts," IEEE Transactions on Medical Imaging, vol. 29, pp. 1676-1687, 92010.

[17] R. J. Schneider, D. P. Perrin, N. V. Vasilyev, G. R. Marx, P. J. del Nido, and R. D. Howe, "Mitral annulus segmentation from four-dimensional ultrasound using a valve state predictor and constrained optical flow," Medical Image Analysis, vol. 16, pp. 497-504, 22012.

[18] A. Tiwari and K. A. Patwardhan, "Mitral valve annulus localization in 3D echocardiography," in Proceedings of the Annual International Conference of the IEEE Engineering in Medicine and Biology Society, EMBS, vol. 2016-October, pp. 1087-1090, IEEE, 82016.

[19] Y. Zheng, A. Barbu, B. Georgescu, M. Scheuering, and D. Comaniciu, "Fast Automatic Heart Chamber Segmentation from 3D CT Data Using Marginal Space Learning and Steerable Features," in 2007 IEEE 11th International Conference on Computer Vision, pp. 1-8, IEEE, 102007.

[20] J. Pedrosa, S. Queiros, J. Vilaca, L. Badano, and J. D’hooge, "Fully automatic assessment of mitral valve morphology from 3D transthoracic echocardiography," in 2018 IEEE International Ultrasonics Symposium (IUS), IEEE, 102018.

[21] R. Hahn, T. Abraham, M. Adams, C. Bruce, K. Glas, R. Lang, S. Reeves, J. Shanewise, S. Siu, W. Stewart, and M. Picard, "Guidelines for performing a comprehensive transesophageal echocardiographic examination: Recommendations from the american society of echocardiography and the society of cardiovascular anesthesiologists," Anesthesia and Analgesia, vol. 118, pp. 21-68, 12014.

[22] A. Vegas, "Three-dimensional transesophageal echocardiography: Principles and clinical applications," Ann Card Anaesth, vol. 19, pp. S35S43, 102016.

[23] O. Ronneberger, P. Fischer, and T. Brox, "U-Net: Convolutional Networks for Biomedical Image Segmentation,” arXiv e-prints, 52015.

[24] O. Chapelle and M. Wu, "Gradient descent optimization of smoothed information retrieval metrics," Information Retrieval, vol. 13, pp. 216235, 62010.

[25] D. C. Luvizon, H. Tabia, and D. Picard, "Human Pose Regression by Combining Indirect Part Detection and Contextual Information," arXiv e-prints, 102017.

[26] A. Nibali, Z. He, S. Morgan, and L. Prendergast, "Numerical coordinate regression with convolutional neural networks," arXiv e-prints, 12018.

[27] G. Dwivedi, G. Mahadevan, D. Jimenez, M. Frenneaux, and R. P. Steeds, "Reference values for mitral and tricuspid annular dimensions using two-dimensional echocardiography.," Echo research and practice, vol. 1, pp. 43-50, 122014.

[28] K. He, X. Zhang, S. Ren, and J. Sun, "Delving deep into rectifiers: Surpassing human-level performance on imagenet classification," in 2015 IEEE International Conference on Computer Vision (ICCV), pp. 10261034, 122015.

[29] D. P. Kingma and J. Ba, "Adam: A method for stochastic optimization," in 3rd International Conference on Learning Representations, ICLR 2015, San Diego, CA, USA, May 7-9, 2015, Conference Track Proceedings, 2015.

[30] Q. Zheng, H. Delingette, N. Duchateau, and N. Ayache, "3-d consistent and robust segmentation of cardiac images by deep learning with spatial propagation," IEEE Transactions on Medical Imaging, vol. 37, pp. 21372148, 92018.

[31] R. Caruana, "Multitask learning," Machine Learning, vol. 28, pp. 41-75, 71997.

[32] P. Moeskops, J. M. Wolterink, B. H. M. van der Velden, K. G. A. Gilhuijs, T. Leiner, M. A. Viergever, and I. Išgum, "Deep learning for multi-task medical image segmentation in multiple modalities," in Medical Image Computing and Computer-Assisted Intervention MICCAI 2016 (S. Ourselin, L. Joskowicz, M. R. Sabuncu, G. Unal, and W. Wells, eds.), (Cham), pp. 478-486, Springer International Publishing, 2016. 